\title{
Cultural misconceptions and associated depression in Dhat syndrome
}

\author{
Neena Sanjiv Sawant, Anand Nath
}

\section{Background}

Dhat syndrome or semen-loss anxiety is characterized by vague somatic symptoms of fatigue, weakness, anxiety and loss of appetite. Sufferers experience guilt attributed to semen loss through nocturnal emissions, urine and masturbation resulting in hypochondriasis, anxiety and depressive symptoms.

Aims

The study aims to evaluate the presence of depression among patients with Dhat syndrome and compare beliefs on issues related to sexuality between patients with Dhat syndrome and a control group.

\section{Methods}

Thirty two patients with Dhat Syndrome attending the psychiatry clinic of a general municipal hospital in India and 33 healthy males accompanying other patients to the clinic were administered a questionnaire regarding sexual myths and misconceptions. Depression was assessed using Beck's depression inventory (BDI).

\section{Results}

All patients with Dhat syndrome were diagnosed as having depression on the BDI total score whereas only $30 \%$ of the controls fulfilled criteria for depression. There was a significant difference in the mean BDI scores between the two groups. Myths and misconceptions on sexual issues were identified in both cases and controls, though they were less common in the control group.

Conclusion

The findings suggest a strong association between Dhat syndrome and depression. There is a need to assess depressive symptoms in patients with Dhat syndrome. The findings also suggest a need to educate the public regarding issues related to sexuality and the Dhat syndrome.

SL J Psychiatry 2012; 3 (1):17-20

\section{Introduction}

Dhat syndrome is considered as an "exotic neurosis of the orient" and is a culture-bound neurotic disorder seen in the Indian subcontinent (1). It is characterized primarily by complaints of loss of semen through urine, nocturnal emissions or masturbation, accompanied by vague symptoms of weakness, fatigue, palpitation and sleeplessness. The condition has no organic aetiology. It may sometimes be associated with sexual dysfunctions such as erectile dysfunction and premature ejaculation and psychiatric illnesses such as depression, anxiety states or phobias (2).

In traditional Hindu writings in upanishads, semen is described as a 'vital fluid'. The discharge of this 'vital fluid', either through sex or masturbation, is associated with marked feelings of anxiety and dysphoria. Often the patient describes the loss of a whitish fluid while passing urine. Marked feelings of guilt may be associated with what the patient assumes as 'excessive' masturbation" (3). However, the complaint of Dhat syndrome is not confined to Hindus, but is widespread among all communities of the Indian subcontinent. It has been reported among Sikhs in Punjab, Buddhists in Sri Lanka and Muslims in Pakistan (4).

A large proportion of the general public from all socioeconomic classes in the Indian subcontinent believes that semen loss is harmful. Seminal fluid is considered an elixir of life both in the physical and in the mystical sense. Its preservation guarantees health, longevity, and supernatural powers. The susceptible individual reacts to the prevalent belief system and to the fears of semen loss (5).

This belief is more frequent in young, single, less educated males from the lower socioeconomic classes (6). The clinical picture includes severe anxiety and hypochondriasis. The patient is preoccupied with the excessive loss of semen by nocturnal emissions. Premature ejaculation and erectile dysfunction are also common. Other somatic symptoms like weakness, easy fatigability, palpitations, insomnia, low mood, guilt and anxiety are often present. Males sometimes report a subjective feeling that their penis has shortened $(7,8,9)$.

In cultures where open discussion about sexual issues is taboo and fears about masturbation exist, the urogenital system is likely to be the focus of preoccupation. . Under stress, persons predisposed to amplification of somatic symptoms and anxieties related to health may focus attention on physiological changes such as turbidity of urine and tiredness, and misattribute them to loss of semen in the light of widely prevalent beliefs. These beliefs may then be confirmed by peers and other lay sources as well as by local practitioners subscribing to similar models.

In India, discussions related to sex are still a taboo and sex education is not imparted in schools. Hence adolescents obtain information related to sexuality from peers which may lead to misconceptions. This study was undertaken to understand the perceptions of 
patients with Dhat syndrome, assess the presence of depression in them and to also determine the various cultural beliefs that could be associated with semen loss, masturbation and penis size

\section{Method}

All patients presenting to the psychiatry clinic in a general municipal hospital in India were screened for their chief complaints and all those who had a primary complaint of passage of semen were referred to the researcher. Patients having sexual dysfunctions such as erectile dysfunction, premature ejaculation associated with the complaint of passage of semen were excluded from the study. Each patient was then interviewed and a diagnosis of Dhat syndrome was considered, based on the International Classification of Diseases (ICD)-10 clinical criteria (10). Thirty two fulfilled the criteria. The control group consisted of 33 healthy males randomly selected from those accompanying patients attending the clinic and who were not seeking any psychiatric help. For both groups, only those in the age group of 18-45 years and were competent to provide written consent were included in the study. Because misconceptions about sexual issues in commoner among the young, the study selected only those aged over 18 years. Persons aged more than 45 years and those with any associated medical or surgical co-morbidity, intellectual disability or substance use disorders were excluded from the study.

The study was approved by the Ethics Review Committee. All subjects were explained about the nature of study and its applications and informed consent was obtained.

\section{Outcome measures}

A study specific questionnaire was used to assess sociodemographic details, utilising the Kuppuswamy scale. It also contained questions pertaining to the aims and objectives of the study (11). A questionnaire based on prevailing myths among males about masturbation, penis, semen, circumcision and vasectomy in India was designed by the researchers and administered to all subjects. The questions were rated on a 3 point Likert scale (Yes $=1$, Not sure $=2$ and $\mathrm{No}=3$ ).

The Beck's depression inventory (BDI) was used to assess depression in the study sample. Both study specific tools were translated to Hindi and Marathi and were validated.

\section{Results}

The mean age of the cases and controls were 25.34 years and 24.06 years respectively. In both groups the majority were Hindus and had a primary or secondary education. In both groups $20(60 \%)$ were unmarried. Among those who were married, 11(78.57\%) were living away from the wife among the cases as compared to $5(38.46 \%)$ subjects in the control group. These persons were working in a major city and their wives were resident in the villages. Twenty two subjects each in the case and control groups $(68.7 \% \& 66.66 \%$ respectively) belonged to the lower socioeconomic group and the remainder to the lower- middle socioeconomic group as designated in the Kuppuswamy scale.
Twenty seven $(81.81 \%)$ patients among the cases and $24(72.72 \%)$ of the control group said they masturbated currently. Exposure to Commercial sex workers was reported by $12(36.36 \%)$ patients among the cases and $8(24.24 \%)$ in the control group.

All the patients with Dhat syndrome were diagnosed as having depression on the BDI. In this group, two $(6.06 \%)$ had borderline clinical depression (cut-off score: 17-20), 13(39.39\%) had moderate depression (cut-off score 21-30), 13(39.39\%) had severe depression (cut-off score: $31-40)$ and $5(15.15 \%$ ) had extreme depression (cut-off score: over 40). Ten persons $(30.3 \%)$ in the control groupware diagnosed as having depression on the BDI. Among them 5(50\%) had borderline clinical depression, 3(30\%) had moderate depression and 2(20\%) had severe depression. (Table1 and 2).

The cases had a significantly higher mean BDI score (32.0) compared to the control group (14.9) (t test 7.57, $\mathrm{p}<0.001)$.

\section{Myths and Misconception}

\section{Masturbation}

Twenty six $(81.2 \%)$ of the cases and $25(75.5 \%)$ of the controls believed that masturbation caused physical and mental deterioration and led to physical exhaustion and weakness. Persons in both groups believed that semen was precious and should not be'wasted' by masturbation as masturbation causes loss of vital elements and affected reproductive capacity.

Sixteen $(50 \%)$ of the cases and $23(69.6 \%)$ of the controls believed that married people should not masturbate. The majority of the cases but not the control group believed that masturbation causes erectile dysfunction due to 'pressure on nerves' and increased the risk of contracting HIV/AIDS. The view that masturbation leads to acne in adolescents and that the penis becomes curved by engaging in masturbation was held by $22(66.6 \%)$ of the controls but none in the case group.

\section{Semen}

Twenty six (81.2\%) of the cases and 30 (90.9\%) of the controls believed that repeated emissions had adverse effect on physical health, frequent emissions made semen thin and caused loss of vigor. Semen was considered precious by all and $23(71.8 \%)$ of the cases and $26(78.7 \%$ ) of the controls believed that spermatorrhoea was a serious condition. .

Table 1 - Severity of Depression according to BDI scores
\begin{tabular}{|ll|l|}
\hline Severity of depression & Cases & Controls \\
(BDI score range) & $\mathrm{N}(\%)$ & $\mathrm{N}(\%)$ \\
\hline Borderline (17-20) & $2(6.06 \%)$ & $5(50 \%)$ \\
Moderate (21-30) & $13(39.39 \%)$ & $3(30 \%)$ \\
Severe (31-40) & $13(39.39 \%)$ & $2(20 \%)$ \\
Extreme $(>40)$ & $5(15.15 \%)$ & $0(0 \%)$ \\
\hline
\end{tabular}




\section{Penis}

Twenty three $23(71.8 \%)$ of the cases and $17(51.5 \%)$ of the controls believed that the shape or curvature of the penis affected sexual pleasure .Twenty five (78.1\%) of the cases and $23(69.6 \%)$ of the controls felt that the penis should remain erect for long periods during sexual activity. Sixteen $16(50 \%)$ of the cases believed that penetration was easier with a large penis and $22(68.7 \%)$ of them felt that consuming certain foods such as onions, meat, and fish could lead to a 'better' erection, contrary to the views of the control group. The majority of the cases and controls did not believe that a small penis affected procreation, that the use of tight underwear reduced the size of the penis, that repeated intercourse increased the size of the penis or that muscular people had large penises.

\section{Vasectomy}

In the control group $17(51.5 \%)$ believed vasectomy reduced sexual drive and $23(69.6 \%)$ felt that it decreased sexual pleasure but the majority of the cases did not endorse these beliefs.

\section{Discussion}

In our study sample, although $80 \%$ of the cases and controls were literate and $50 \%$ of them had secondary or higher education, myths about sexual issues were common. In India there is a lack of proper sex education in schools and colleges. Similar results were found by Khan and Singh $(6,13)$. Misconceptions were more prevalent among unmarried males and amongst the married males who originated from small villages and were living away from their wives. This could be attributed to decreased frequency of intercourse, leading to nocturnal emissions and passage of semen at times other than intercourse. These findings are consistent with research done in other cultures $(1,13)$. De Silva and Dissanayake and Bhatia and Malik, found a preponderance of unmarried males among those with Dhat syndrome which could be due to the fact that they were worried about the implications of semen loss for their future marital life $(8,14)$. Among this group, marriage may have been delayed because of the prevailing belief that they would have to rid themselves of this affliction if they were to enjoy a normal healthy, sexual life.

In our study, Dhat syndrome was strongly associated with depressive symptoms which is similar to the findings of other researchers. Though depressive symptoms were evident on clinical interview, the BDI was used to diagnose and rate the severity of the symptoms. This is a limitation of the study as this could have led to an over-diagnosis of depression.

In our study all the cases fulfilled criteria for depression based on BDI scores $(1,15)$. Only 18 in the case group had severe or extreme depression. Cases had a significantly higher BDI score than controls. Dhikavin his study found depression in 20 out of 30 patients with Dhat syndrome (3). Chadda and Bhatia, in their respective studies, found the prevalence of depression in those with Dhat syndrome to be $40.3 \%$ and $39.5 \%$ respectively. These findings indicate that there is a need to assess depressive symptoms in patients with Dhat syndrome.

There was a high prevalence of myths and misconceptions amongst the cases as compared to controls. Masturbation-related myths were most frequent (17). It was believed that loss of even one drop of semen would cause the male to experience weight loss, weakening of eye sight, watery consistency of semen, reduction in quantity of semen, extreme weakness and failure to become a father. The males in both the groups had little knowledge about vasectomy and its effect on sexuality.

These findings are similar to those reported by Chaddha and Ahuja (1). The ancient belief about semen conservation has a long philosophical history in Europe and Asia. In Sanskrit, semen is equated with sukra, the life force (18). In the Indian Ayurvedic system of medicine, sukra is considered essential for health and it is believed that loss of semen in any form leads to depletion of physical and mental energy (1). The Ayurvedic system proposes that one drop of semen is equivalent to of 100 drops of blood and that one drop of blood is derived from minerals and nutrition ingested by the male. Because of this semen takes long to 'regenerate'. Hence Ayurveda advises celibacy to preserve male vitality (18).

These myths and misconceptions which are deeply rooted in Indian culture are passed from generation to generation. Due to the lack of proper information and lack of open communication between parents and children, the only source of knowledge for many remain their peers, who are equally ignorant about the subject, and this leads to widespread misconceptions. Many people consult unqualified practitioners who reinforce their ignorance. Khan reported that a majority of patients with sexual problems resort to alternative medicines and treatment methods (6).

These findings reinforce the need to integrate sex education to the curriculum at high school and college levels. Health care providers could also educate clients on reproduction and sexuality. Health workers engaged in alternative medicine could also contribute since many patients with sexual difficulties consult practitioners of alternative medicine. Public awareness programs on sexual issues may also be beneficial.

\section{Acknowledgments}

We thank the Indian Council of Medical Research for financial assistance to conduct the study.

\section{Declaration of interest}

None

\section{References}

1. Chadda RK, Ahuja N. Dhat syndrome: A sex neurosis of the Indian subcontinent. British Journal of Psychiatry 1990;156:577 -579. 
2. Sumathipala A, Siribaddana SH, Bhugra D. Culture bound syndromes: The story of Dhat syndrome. Br J Psychiatry 2004;184:200-9.

3. Dhikav V, Aggarwal N, Gupta S, Jadhavi R, Singh K . Depression in Dhat syndrome. J Sex Med 2008;5 (4): $841-4$.

4. Mumford DB. The 'Dhat syndrome': a culturally determined symptom of depression. Acta Psychiatr Scand 1996;94(3):163-7.

5. Malhotra HK, Wig NN. Dhat syndrome: a culturebound sex neurosis of the orient. Arch Sex Behav 1975;4(5):519-28.

6. Khan N. Dhat syndrome in relation to demographic characteristics. Indian J Psychiatry 2005;47:54-7

7. Behere PB, Nataraj GS. Dhat syndrome: the phenomenology of a culture bound sex neurosis of the Orient. Indian Journal of Psychiatry 1984;26:76-78

8. Bhatia MS, Malik SC. Dhat syndrome- A useful diagnostic entity in Indian culture. British Journal of Psychiatry 1991;159: 691-695

9. Chadha RK. Dhat syndrome: is it a distinct clinical entity? Acta Psychiatrica Scandinavica 1995;91(2):136 $-139$

10. World Health Organization. International Statistical Classification of Diseases and Related Health Problems. 10th rev. Geneva: World Health Organization: 1992

11. Kuppuswamy B. Manual of socioeconomic status (urban). Delhi; Manasayan,1981.

12. Beck AT, Ward C, Mendelson M. Beck Depression Inventory. Arch Gen Psychiatry 1961;4: 561-571.

13. Singh G. Dhat syndrome revisted. Indian J Psychiatry 1985;27:119-22.

14. De Silva P, Dissanayake SAW. The loss of semen syndrome in Sri Lanka: A clinical study. Sexual and Marital Therapy 1989;4:195-204

15. Bhatia MS, Choudhary S. Dhat syndrome: A culture bound sexual neurosis. Indian J Med Sci. 1998;52:30-5.

16. Bhatia MS. An analysis of 60 cases of culture bound syndromes. Indian J Med Sci. 1999;53(4):149-52.

17. Williams W. Sexual myths in the male. Aust Fam Physician. 1990;19(6):857-60, 863.

18. Money J, Prakasam K, Joshi V. Semen conservation doctrine from ancient ayurvedic to modern sexological theory. Am J Psychother 1991;45:9-14
Neena Sanjiv Sawant,

Professor ( Addl), Dept of Psychiatry

Seth G.S. Medical College and KEM Hospital,

Mumbai - 400012, India.

Anand Nath

Seth G.S. Medical College and KEM Hospital,

Mumbai - 400012,India.

Corresponding author

Neena Sanjiv Sawant

E mail: drneenas@yahoo.com 